\title{
Associations of Motor Developmental Risks with the Socioeconomic Status of Preschool Children in North-Eastern Germany
}

\author{
Annika Gottschling-Lang, Marco Franze, and Wolfgang Hoffmann \\ Institute for Community Medicine, Section Epidemiology of Health Care and Community Health, \\ University Medicine Greifswald, 17487 Greifswald, Germany \\ Correspondence should be addressed to Annika Gottschling-Lang; annika.gottschling@uni-greifswald.de
}

Received 17 April 2013; Accepted 20 November 2013

Academic Editor: Xinyin Chen

Copyright (C) 2013 Annika Gottschling-Lang et al. This is an open access article distributed under the Creative Commons Attribution License, which permits unrestricted use, distribution, and reproduction in any medium, provided the original work is properly cited.

\begin{abstract}
Aims. The study is part of the pilot project "children in preschools" and aims to detect developmental risks of preschool children in the context of their socioeconomic status (SES) as a base to initiate individual intervention strategies. Methods. The "Dortmund Developmental Screening for the Kindergarten" was used in 12 preschools in Mecklenburg-Western Pomerania (MWP) to detect early developmental risks in children aged 3 to 6 years $(n=870)$. Socioeconomic data from $n=530$ parents were collected by a standardised questionnaire. Results. Significant differences between the SES groups were identified especially in the field of fine motor skills $(P<0.05)$. In gross motor development differences were not statistically significant. Prevalence rate of fine motor developmental risks ranges from $1.7 \%$ to $20.9 \%$; the rate of gross motor developmental risks tops out at $14.4 \%$. The prevalence rates are associated with age and sex. Conclusions. Fine motor skills in 3-6 years old preschool children are significantly associated with the socioeconomic status. In gross motor skills an association could not be identified. In this study, motor development was more affected by sex than by SES.
\end{abstract}

\section{Introduction}

The health situation of children and youth is currently changing on national and European level, from acute to more chronic diseases and from somatic to mental disorders (new morbidity) [1]. The prevalence rate of developmental disorders can reach high. In particular, the KiGGS studyThe German Health Interview and Examination Survey for Children and Adolescents-found that $13.1 \%$ of girls and $18.4 \%$ of boys aged 3 to 6 years have behavioural abnormalities $[2,3]$. In several studies there is evidence to suggest a relation between the socioeconomic status (SES) and children's development: children with a lower SES are more affected by developmental delays $[4,5]$.

Such developmental disorders are negative factors for children to grow up healthily. Especially motor developmental delays are often associated with an inactive lifestyle and children are affected additionally by overweight or obesity
[6]. Throughout the lifespan, overweight and the lack of exercise are main risk factors for cardiovascular diseases (e.g., arteriosclerosis), problems in the musculoskeletal system or some metabolic diseases (e.g., diabetes mellitus). One problem is that children from low-income households are more inclined to be overweight and suffer from lack of exercise $[7,8]$. So, it can be assumed that these children are also more affected by motor developmental risks.

The federal state Mecklenburg-Western Pomerania (MWP) - located in the north-eastern part of Germany-is affected by a high unemployment rate (MWP 2008: 15.5\%, whole Germany 2008: 7.8\%), a weak economic development, and a low average annual income (average annual income per inhabitant in MWP: 14,610 euro; national average: 18,411 euro) $[9,10]$.

School enrolment examinations show a high prevalence rate of motor developmental delays (20.3\%) [11]. Furthermore, $12.4 \%$ of children aged 3 to 6 years are overweight 
and $5.5 \%$ have obesity. These prevalence rates are above the national average. The proportion of overweight of 36 -year-olds in Germany is 9.0\%; the proportion of obesity in this age group is $2.9 \%$ [12]. Several studies suggest that social disadvantages have a negative effect on children's development and their health state [13-15]. In contrast, the state of motor development is often advanced if children belong to a higher social class [16-18].

Taking into account socioeconomic data and rates of developmental delays in MWP, it can be expected that in this federal state there is also a strong association between both predictors. So there is an urgent need to detect developmental risks and to initiate individual intervention strategies early in time. It seems probable that undetected developmental risks will become chronic delays. As fine and gross motor skills are positively associated with cognitive abilities [19] and with the health situation in later life, tailored interventions should be applied already in preschool age. Because of the high utilisation rate of preschools in MWP-93.1\% of the children between 3 and 6 years visit this institution although it is not obligatory [20] - preschools are well-suited for a settingorientated intervention.

The aim of the present study is to analyse the prevalence rate of developmental risks and the differences between the state of fine and gross motor development related to the SES of preschool children in MWP. It might be assumed that increasing motor skills are associated with increasing SES. Furthermore, possible differences should be investigated by sex because it seems to be possible that girls and boys are influenced by SES in a different way.

Results should provide further indications for better intervention strategies to avoid developmental delays as a possible negative effect of social inequality.

\section{Methods}

2.1. The Study "KiK". The pilot project "KiK" (in German "Kinder in Kitas" means "children in preschools") is designed as a cluster-randomised, controlled, prospective study.

12 preschools selected by youth welfare offices in MWP, participated in this study. The preschools are located in cities (Wismar, Greifswald, and Stralsund) and in rural areas (administrative districts Eastern Pomerania, Demmin and Uecker-Randow). From each areas two preschools were recruited by the youth welfare offices. These preschools were randomised to an intervention group and a control group.

2.2. The Dortmund Developmental Screening for Preschools. The Dortmund Developmental Screening for Preschools (Dortmunder Entwicklungsscreening für den Kindergarten DESK 3-6) was used to detect early developmental risks [21]. It is a standardized, valid, and reliable instrument which adheres to scientific and practical conditions [22, 23].

The screening includes active exercises and monitoring of motor, linguistic, and social development. Also, a result for the overall development is given. The items are age-based and vary in three age groups (3-year-olds, 4-year-olds, and 5- to 6 -year-olds).
In the following analysis fine and gross motor skills are in the focus of interests. Examples for the tasks concerning fine and gross motor skills are as follows:

Buttoning or unbuttoning a button is one task in the field of fine motor skills for 3- and 4-year-olds. Painting a human being consisting of six parts is a task-example for children aged 4 to 6 years. With these tasks competences like eye-hand-coordination and the precision of hand-movement should be tested.

In the field of gross motor skills (coordination, balance control) 3- and 4-year-olds, for example, have to catch a ball from a distance of two meters. Also, children aged 4 to 6 years should be able to show toe walking and heel walking backwards.

Results of the screening are stanine values (standard nine values) between 1 and 9 for each field of development (motor, linguistic/cognitive, and social) and for the total development. A value of " 1 " indicates a reasonable suspicion of developmental risks. A stanine value of " 2 " stands for an inconclusive screening-result where a definite decision about developmental risks is not possible. The screening has to be replicated later on. Stanine values between 3 and 9 indicates no suspicion for developmental delays. Higher stanine values are associated with a more advanced level.

The screening should be conducted by the familiar nursery-school teachers. In this project the nursery-school teachers were given a training in how to perform the developmental screening [24].

2.3. Parental Survey. Data about parents' SES was collected by mail through a standardised questionnaire [25]. The questionnaire included questions about education (e.g., graduation), occupational classification, and monthly income. On the basis of this information the SES (Winkler-Index) was assessed and the respondents were categorised in low, middle, and high SES group [26].

2.4. Statistical Analyses. The analysis of the data was made using PASW (version 18.0).

Data are reported as mean with $95 \%$ confidence interval (CI) and standard deviation (SD). Mean differences of the stanine values and the association of motor development, socioeconomic state, and additional sex were analysed by two-way analysis of variance followed by Scheffe post hoc tests at $P=0.05$.

Two-tailed tests were performed at the 5\% level of significance using $t$-tests for independent samples to assess sex and age differences. Cohen's $d$ effect size was used for the interpretation of the $t$-tests $(.20=$ small, $.50=$ moderate, and $.80=$ large effect) [27].

In the study, only pseudonymised data were processed. Pseudonyms were generated using a hash function.

Declaration of Helsinki. The study was ethically and legally allowed by the local Ethics Committee of the University Medicine Greifswald. 


\section{Results}

Overall, $n=870$ children at the age of three to six years were screened (response rate $=92.4 \%)$. Only $0.5 \%(n=6)$ of parents refused for their children to participate in the developmental screening. The remaining children (5.58\%; $n=66$ ) were out of preschool during the screening.

Children with disabilities or chronic diseases were excluded from the following calculations $(n=32)$. Data records with missing values were also excluded $(n=239)$.

Finally, $n=636$ were included in the study; $53.0 \%(n=$ $337)$ male, $47.0 \%(n=299)$ female. On screening $28.8 \%(n=$ $183)$ of the children were 3 years old, $28.0 \%(n=178) 4$ years old and finally $43.2 \%(n=275) 5$ to 6 years old.

The response rate of the social status questionnaire was $56.3 \%(n=520)$. Of all parents who answered the questions about education, occupational classification, and income, $37.5 \%(n=195)$ belonged to the group with a low SES, $43.8 \%$ ( $n=228)$ to the group with a middle, and $18.7 \%(n=97)$ to the group with a high SES.

A non responder analysis did not show significant differences in the stanine means of children whose parents returned the questionnaires versus children whose parents did not.

The analysis of the relationship between motor development and SES of preschool children included $n=344$ parentchild couples.

The descriptive statistics for the stanine means are presented in Table 1. Tables 2 and 3 show the results of twoway ANOVA. The three SES groups differed significantly with respect to the level of fine motor skills $(P<0.05)$. Scheffe post-hoc test indicated significant differences between children with a low and a high SES $(P<0.05)$ but not between children with a low and a middle or a middle and a high SES $(P=$ n.s. $)$. As well as the SES, sex influenced fine motor skills (Table 2). An interaction between SES and sex did not exist.

Although the stanine-means of gross motor skills increased with socioeconomic status, differences between social classes were not significant $(P=0.078)$. Sex based differences were statistically significant also in the field of gross motor skills (Table 3). Again, an interaction between SES and sex could not be identified.

A two-way ANOVA for each age group, including both girls and boys, suggested no significant associations between motor development and socioeconomic status.

Sex specific analysis (separate two-way analysis for girls and boys with SES group and age group as fixed factors) showed an influence of SES only for girls' fine motor skills (sum of squares $=17.81 ; \mathrm{df}=2$; mean square $=8.90 ; F=$ $3.10 ; P<0.05)$. Scheffe post-hoc test indicated significant differences between girls with a low and a high SES $(P<$ 0.05).

Table 4 shows age and sex-specific stanine means and standard deviations for fine and gross motor skills independent of the SES.

In all age groups, male children had significantly lower stanine means in the development of fine motor skills than female children (all $P$ values at least: $P<0.05$ ). Boys aged
4 to 6 years had also significant lower stanine means for gross motor skills than girls (all $P$-values at least: $P<0.05$ ).

However, the calculated effect size Cohen's $d$ was .34 (fine motor skills) and .20 (gross motor skills) for 3-yearold children, representing small effect sizes. In the group of 4-year-old boys and girls Cohen's $d$ was .52 (fine motor skills), and .38 (gross motor skills) respectively, so a small-tomoderate effect size. Finally, Cohen's $d$ effect size was .82 (fine motor skills) and .52 (gross motor skills) for 5- and 6-year-old children. This implies a moderate-to-large effect.

The results in Table 5 show that the prevalence rate of fine motor developmental risks (stanine-value of "1") was between $1.7 \%$ (5- and 6-year-old girls) and 20.9\% (5- and 6-year-old boys) and varied with age and sex.

The prevalence rate of gross motor developmental risks was between $0.0 \%$ (4 and 5-6-year-old girls) and 14.4\% (3year-old boys). Only girls aged 4,5 , and 6 years were not affected by gross motor developmental risks.

In all age-groups some exercises were performed easily by a high proportion of children, whereas others posed difficulties. In detail, in the field of fine motor skills a high proportion of 3-year-old children were not able to unbutton or button up a button (32.2\%). Furthermore, more than a quarter of all screened 4-year-old children (20.3\%) had problems to paint a picture with several objects (e.g., a picture with a tree, a house, and a sun). Another example for a difficult task in the age-group of 5- to 6-year-old children is the transcription of at least three numbers or letters. $13.6 \%$ of the children did not solve this task. In contrast, for $93.0 \%$ of the children of the same age-group other tasks such as using a rubber were uncomplicated.

In the field of gross motor skills, $23.1 \%$ of the 3 -year-old children were unable to jump on just one foot. Remarkably, $9.5 \%$ of the 5 - and 6-year-old children could not bounce a ball (e.g., a football or volleyball) four times. However, $93.8 \%$ of the 4 -year-old children had no problems to pass five finishing jumps.

\section{Discussion}

A screening like the DESK 3-6 is an adequate opportunity to get a first impression of children's development [22]. However, the results of a screening do not substitute a definite diagnose. If the screening results indicate a developmental risk, for example, a paediatrician should be contacted to perform detailed medical examination/diagnostic and to additionally exclude physical causes for the developmental risk.

The results of this study suggest a considerable percentage of children with developmental risks (fine motor skills up to $20.9 \%$; gross motor skills up to $14.4 \%$ ). Fine motor skills were more affected than gross motor skills.

Based on the suggestion of Cohen [27], in this study the motor development of 3- to 6-year-old preschool children showed only a weak association with socioeconomic status. In literature already effect sizes of .25 are described as significant [28]. Therefore, the association between motor development and SES could also be interpreted as strong. 
TABLE 1: Descriptive statistics for screening results (stanin-means) in fine and gross motor skills differentiated by SES and sex $(n=344)$.

\begin{tabular}{|c|c|c|c|c|c|c|c|}
\hline \multirow{2}{*}{ SES } & \multirow{2}{*}{ Sex } & \multicolumn{3}{|c|}{ Fine motor skills } & \multicolumn{3}{|c|}{ Gross motor skills } \\
\hline & & Mean & Std. Deviation & $N$ & Mean & Std. Deviation & $N$ \\
\hline \multirow{3}{*}{ Low } & Male & 3.20 & 1.67 & 59 & 4.44 & 2.00 & 59 \\
\hline & Female & 4.27 & 1.7 & 64 & 5.09 & 1.91 & 64 \\
\hline & Total & 3.67 & 1.78 & 123 & 4.78 & 1.97 & 123 \\
\hline \multirow{3}{*}{ Medium } & Male & 3.63 & 1.78 & 79 & 4.51 & 2.23 & 79 \\
\hline & Female & 4.71 & 1.68 & 75 & 5.97 & 1.97 & 75 \\
\hline & Total & 4.16 & 1.81 & 154 & 5.22 & 2.23 & 154 \\
\hline \multirow{3}{*}{ High } & Male & 4.09 & 1.77 & 33 & 5.12 & 1.82 & 33 \\
\hline & Female & 5.21 & 1.57 & 34 & 5.56 & 1.73 & 34 \\
\hline & Total & 4.66 & 1.75 & 67 & 5.34 & 1.77 & 67 \\
\hline \multirow{3}{*}{ Total } & Male & 3.57 & 1.76 & 171 & 4.60 & 2.08 & 171 \\
\hline & Female & 4.64 & 1.71 & 173 & 5.57 & 1.93 & 173 \\
\hline & Total & 4.11 & 1.81 & 344 & 5.09 & 2.06 & 344 \\
\hline
\end{tabular}

Stanine-value of 1: reasonable suspicion for developmental risks.

Stanine-value of 2: inconclusive screening-result.

Stanine-value of 3-9: no suspicion for developmental risks.

TABLE 2: Results of two-way ANOVA for comparison mean differences in fine motor skills in low SES group, medium SES group and high SES group and in sex $(n=344)$.

\begin{tabular}{|c|c|c|c|c|c|}
\hline Source & Sum of squares & $\mathrm{df}$ & Mean square & $F$ & $P$ \\
\hline SES & 37.279 & 2 & 18.639 & 6.362 & 0.002 \\
\hline Sex & 89.353 & 1 & 89.353 & 30.499 & 0.000 \\
\hline SES $*$ sex & 0.031 & 2 & 0.016 & 0.005 & 0.995 \\
\hline Error & 990.231 & 338 & 2.930 & & \\
\hline Total & 6938.000 & 344 & & & \\
\hline Corrected total & 1125.802 & 343 & & & \\
\hline
\end{tabular}

TABLE 3: Results of two-way ANOVA for comparison mean differences in gross motor skills in low SES group, medium SES group and high SES group and in sex $(n=344)$.

\begin{tabular}{|c|c|c|c|c|c|}
\hline Source & Sum of squares & $\mathrm{df}$ & Mean square & $F$ & $P$ \\
\hline SES & 20.436 & 2 & 10.218 & 2.574 & 0.078 \\
\hline Sex & 55.307 & 1 & 55.307 & 13.934 & 0.000 \\
\hline SES $*$ sex & 17.331 & 2 & 8.665 & 2.183 & 0.114 \\
\hline Error & 1341.571 & 338 & 3.969 & & \\
\hline Total & 10362.000 & 344 & & & \\
\hline Corrected total & 1459.384 & 343 & & & \\
\hline
\end{tabular}

TABLE 4: Stanine-means and standard deviation (SD) of fine and gross motor skills differentiated between age and sex $(t$-test, $n=636)$.

\begin{tabular}{lccccccccc}
\hline Age & \multicolumn{3}{c}{ 3-year-old } & \multicolumn{3}{c}{ 4-year-old } & \multicolumn{3}{c}{ 5- to 6-year-old } \\
\hline Sex $(n)$ & $\begin{array}{c}\text { Male } \\
(90)\end{array}$ & $\begin{array}{c}\text { Female } \\
(86)\end{array}$ & {$[95 \% \mathrm{CI}]^{1}$} & $\begin{array}{c}\text { Male } \\
(90)\end{array}$ & $\begin{array}{c}\text { Female } \\
(78)\end{array}$ & {$[95 \% \mathrm{CI}]^{1}$} & $\begin{array}{c}\text { Male } \\
(134)\end{array}$ & $\begin{array}{c}\text { Female } \\
(121)\end{array}$ & {$[95 \% \mathrm{CI}]^{1}$} \\
\hline Fine motor & $3.88^{*}$ & $4.52^{*}$ & -1.201 to & $3.88^{* *}$ & $4.83^{* *}$ & -1.512 to & $\begin{array}{c}3.22^{* *} \\
(1.76)\end{array}$ & $\begin{array}{c}4.58^{* *} \\
(1.57)\end{array}$ & -1.768 to \\
skills (SD) & $(1.77)$ & $(1.96)$ & -0.090 & $(1.83)$ & $(1.81)$ & -0.399 & -0.944 \\
\hline Gross motor & 4.48 & 4.92 & -1.101 to & $5.27^{*}$ & $5.86^{*}$ & -1.063 to & $4.59^{* *}$ & $5.60^{* *}$ & -1.498 to \\
skills (SD) & $(2.17)$ & $(2.26)$ & 0.219 & $(1.69)$ & $(1.36)$ & -0.121 & $(2.10)$ & $(1.80)$ & -1.949 \\
\hline
\end{tabular}

Significant sex differences $\left(t\right.$-test): ${ }^{*} P<0.05 ;{ }^{* *} P<0.01$.

${ }^{1}$ Confidence Interval for the mean difference between two independent proportions (boys versus girls). 
Table 5: Prevalence of fine and gross motor developmental risks ( tanine-value $=1 ; n=636$ ).

\begin{tabular}{|c|c|c|c|c|c|c|}
\hline \multirow[b]{2}{*}{ Age } & \multicolumn{3}{|c|}{ Fine motor skills } & \multicolumn{3}{|c|}{ Gross motor skills } \\
\hline & 3 years $^{1}$ & 4 years $^{2}$ & $5 / 6$ years $^{3}$ & 3 years $^{1}$ & 4 years $^{2}$ & $5 / 6$ years $^{3}$ \\
\hline Female & $10.5 \%(n=9)$ & $2.6 \%(n=2)$ & $1.7 \%(n=2)$ & $7.0 \%(n=6)$ & $0.0 \%(n=0)$ & $0.0 \%(0)$ \\
\hline Male & $11.1 \%(n=10)$ & $12.2 \%(n=11)$ & $20.9 \%(n=28)$ & $14.4 \%(n=13)$ & $2.2 \%(n=2)$ & $6.7 \%(n=9)$ \\
\hline
\end{tabular}

${ }^{1}$ Female: $n=86$, male: $n=90$.

${ }^{2}$ Female: $n=78$, male: $n=90$.

${ }^{3}$ Female: $n=121$, male: $n=134$.

Significant differences in the development were identified between children with a low and a high SES (fine motor skills). Gross motor skills were not affected by SES.

Generally, in this study motor development was more affected by sex than by SES, which was the priority focus.

These findings might result from the fact that perhaps interests of girls in this age group (e.g., handicraft work or crayoning) are more dependent on the provision of craft materials and parents' "collaboration," which are associated with socioeconomic status $[29,30]$ than the interests of male children (e.g., outdoor activities). The age group had no effect on a different development in context of the socioeconomic status.

An interesting finding was an increasing statistical power of sex differences in stanine means with increasing of age. These significant sex differences suggest the need for sexspecific interventions or advancement for boys.

One limitation of this study is the parental response rate of only $56.3 \%$ with regard to the socioeconomic questionnaire. A reason for this might be the sensitive information on family income.

Concerning the responders in $8.1 \%(n=42)$ of the cases it was not possible to generate parent-child-couples via the hash codes. A different spelling of children's first name by parents and nursery-school teachers, resulting in a different hash code for the same child, could be identified as a reason for this problem.

A positive selection of children with higher staninescores can be ruled out because the non-responder-analysis did not show significant differences in the stanine-means of children whose parents returned the questionnaires versus children whose parents did not.

Preventive interventions are necessary for all preschool children because the socioeconomic status cannot be the only reason for high prevalence rates in motor developmental risks. In this age, primary motor skills according to the normal physical development improve in quality and quantity [31]. However, this might depend on the environment and functioning family relations. Therefore, the influence of free-time activities and the utilisation of media should be additionally analysed.

Because of the high utilisation rate of preschools especially in Mecklenburg-Western Pomerania (93.1\%, 2008 [20]), preschools are an important institution to offer programmes and activities for the promotion of competences independent of children's socioeconomic background. This would provide an important contribution to achieve equal opportunities in motor development before school enrolment.
Perspective. Future studies should further investigate the reasons for the high rates of suspected motor developmental risks. In preschools, evidence based programmes to advance fine and gross motor skills have to be implemented and parents have to get more information about their own important active role regarding the motor development of their children.

\section{Conflict of Interests}

The authors declare that there is no conflict of interests.

\section{Acknowledgments}

The authors would like to thank all preschools participating in the pilot project "Children in Preschools" including all nursery-school teachers, children, and parents. This study was supported by the Ministry of Social Affairs and Health Mecklenburg-Western Pomerania (grant numbers IX220 a, LJA-220 b).

\section{References}

[1] J. S. Palfrey, T. F. Tonniges, M. Green, and J. Richmond, "Introduction: addressing the millennial morbidity: the context of community pediatrics," Pediatrics, vol. 115 , no. 4, pp. 11211123, 2005.

[2] H. Hölling, M. Erhart, U. Ravens-Sieberer, and R. Schlack, "Behavioural problems in children and adolescents: first results from the German Health Interview and Examination Survey for Children and Adolescents (KiGGS)," BundesgesundheitsblattGesundheitsforschung-Gesundheitsschutz, vol. 50, no. 5-6, pp. 784-793, 2007.

[3] U. Ravens-Sieberer, N. Wille, S. Bettge, and M. Erhart, "Mental health of children and adolescents in Germany: results from the BELLA study within the German Health Interview and Examination Survey for Children and Adolescents (KiGGS)," Bundesgesundheitsblatt-GesundheitsforschungGesundheitsschutz, vol. 50, no. 5-6, pp. 871-878, 2007.

[4] B. I. Avan and B. Kirkwood, "Role of neighbourhoods in child growth and development: does 'place' matter?" Social Science and Medicine, vol. 71, no. 1, pp. 102-109, 2010.

[5] D. Grant and S. Kurosky, "Trends in the health of young children in California," Policy Brief, no. 2008-3, pp. 1-8, 2008.

[6] M. Slining, L. S. Adair, B. D. Goldman, J. B. Borja, and M. Bentley, "Infant overweight is associated with delayed motor development," Journal of Pediatrics, vol. 157, no. 1, pp. 20-25, 2010. 
[7] C. F. Rosaneli, F. Auler, C. B. Manfrinato et al., "Evaluation of the prevalence and nutritional and social determinants of overweight in a population of schoolchildren: a cross-sectional analysis of 5, 037 children," Revista da Associação Médica Brasileira, vol. 58, no. 4, pp. 472-476, 2012.

[8] N. Spencer, T. M. Thanh, and S. Louise, "Low income/socioeconomic status in early childhood and physical health in later childhood/adolescence: a systematic review," Maternal and Child Health Journal, vol. 17, no. 3, pp. 424-431, 2013.

[9] Federal Statistical Office and the statistical Offices of the Länder, Arbeitslose und Arbeitslosenquote im Jahresdurchschnitt, Schwerin, Germany, 2011, http://www.mvnet.de/inmv/landmv/stala/sis/tabelle.php?\&id=3678.

[10] Statistical Office of Mecklenburg-Western Pomerania, Verfügbares Einkommen je Einwohner erreicht im Jahr 2007 79, 4 Prozent des Bundesdurchschnitts, Schwerin, Germany, 2011, http://www.statistik-mv.de/cms2/STAM_prod/STAM/de/ep/ Presseinformationen/index.jsp?\&pid=12478.

[11] Ministry of Social Policy and Health Mecklenburg-Western Pomerania, Befunde bei Einschulungsuntersuchungen Mecklenburg-Vorpommern, in \% der Untersuchten. Schuljahr 200304 bis 2008-09, Schwerin, Germany, 2011, http://www.regierungmv.de/cms2/Regierungsportal_prod/Regierungsportal/de/ sm/Themen/Gesundheit_und_Arbeitsschutz/Gesundheitsberichterstattung/_Indikatoren/Download_der_Gesundheitsindikatoren_MV.

[12] B.-M. Kurth and A. S. Rosario, "The prevalence of overweight and obese children and adolescents living in Germany: results of the German Health Interview and Examination Survey for Children and Adolescents (KiGGS)," BundesgesundheitsblattGesundheitsforschung-Gesundheitsschutz, vol. 50, no. 5-6, pp. 736-743, 2007.

[13] M. Lange, P. Kamtsiuris, C. Lange, A. S. Rosario, H. Stolzenberg, and T. Lampert, "Sociodemographic characteristics in the German Health Interview and Examination Survey for Children and Adolescents (KiGGS): operationalisation and public health significance, taking as an example the assessment of general state of health," Bundesgesundheitsblatt-GesundheitsforschungGesundheitsschutz, vol. 50, no. 5-6, pp. 578-589, 2007.

[14] M. Quell and L. Sattel, "Untersuchungen zum Zusammenhang zwischen sensomotorischer Entwicklung von Kinder der Primarstufe und der Zugehörigkeit ihrer Eltern zu verschiedenen sozialen Schichten," Sportunterricht, vol. 25, pp. 293-299, 1976.

[15] C. Scheffler, K. Ketelhut, and U. Morgenstern, "Physical and motor development of children with different social origins," Anthropologischer Anzeiger, vol. 62, no. 4, pp. 421-428, 2004.

[16] H. Krombholz, "Physical performance in relation to age, sex, social class and sports activities in kindergarten and elementary school," Perceptual and Motor Skills, vol. 84, no. 3, pp. 1168-1170, 1997.

[17] M. McPhillips and J.-A. Jordan-Black, "The effect of social disadvantage on motor development in young children: a comparative study," Journal of Child Psychology and Psychiatry and Allied Disciplines, vol. 48, no. 12, pp. 1214-1222, 2007.

[18] A. Starker, T. Lampert, A. Worth, J. Oberger, H. Kahl, and K. Bös, "Motor fitness: results of the German health interview and examination survey for children and adolescents (KiGGS)," Bundesgesundheitsblatt-GesundheitsforschungGesundheitsschutz, vol. 50, no. 5-6, pp. 775-783, 2007.

[19] J. P. Piek, L. Dawson, L. M. Smith, and N. Gasson, "The role of early fine and gross motor development on later motor and cognitive ability," Human Movement Science, vol. 27, no. 5, pp. 668-681, 2008.

[20] Länderreport Frühkindliche Bildungssysteme, B. Stiftung, Ed., Bertelsmann Stiftung, Gütersloh, Germany, 2008.

[21] H. Tröster, D. Flender, and D. Reineke, DESK 3-6. Dortmunder Entwicklungsscreening für den Kindergarten, Hogrefe, Göttingen, Germany, 2004.

[22] T. Kliche, C. Wittenborn, and U. Koch, "The potential performance of developmental screenings in kindergarten. Characteristics and circulation of currently available instruments in Germany," Praxis der Kinderpsychologie und Kinderpsychiatrie, vol. 58, no. 6, pp. 419-433, 2009.

[23] H. Tröster, J. Flender, and D. Reineke, "Predictive validity of the Dortmunder Entwicklungsscreening für den Kindergarten (DESK 3-6)," Diagnostica, vol. 57, no. 4, pp. 201-211, 2011.

[24] M. Franze, A. Gottschling, and W. Hoffmann, "The "Dortmund Developmental Screening for Preschools" (DESK 36) as the basis for developmental promotion in preschools in Mecklenburg-West Pomerania. First results of the pilot project "Children in Preschools" referring to the acceptance of DESK 3-6 by preschool teachers," BundesgesundheitsblattGesundheitsforschung-Gesundheitsschutz, vol. 53, no. 12, pp. 1290-1297, 2010.

[25] Robert Koch-Institut, Ed., Studie Zur GesUndheit Von Kindern Und Jugendlichen in Deutschland. Eltern. 3-6, Robert KochInstitut, Berlin, Germany, 2003.

[26] J. Winkler, "Die Messung des sozialen Status mit Hilfe eines Index in den Gesundheitssurveys der DHP," in Messung Soziodemographischer Merkmale in Der Epidemiologie, W. Ahrens, B. M. Bellach, and K. H. Jäckel, Eds., pp. 69-86, MMV Medizin, München, Germany, 1998.

[27] J. Cohen, Statistical Power Analysis for the Behavioral Sciences, Lawrence Erlbaum Associates, Hillsdale, UK, 2nd edition, 1988.

[28] R. E. Slavin and O. S. Fashola, Show Me the Evidence: Proven and Promising Programs for America's Schools, Corwin Press, Thousand Oaks, Calif, USA, 1998.

[29] R. Brockman, R. Jago, K. R. Fox, J. L. Thompson, K. Cartwright, and A. S. Page, "get off the sofa and go and play': family and socioeconomic influences on the physical activity of 10-11 year old children," BMC Public Health, vol. 9, article 253, 2009.

[30] J. Ziviani, D. Wadley, H. Ward, D. Macdonald, D. Jenkins, and S. Rodger, "A place to play: socioeconomic and spatial factors in children's physical activity," Australian Occupational Therapy Journal, vol. 55, no. 1, pp. 2-11, 2008.

[31] K. Roth and C. Roth, "Entwicklung motorischer Fertigkeiten," in Handbuch Motorische Entwicklung, J. Baur, K. Bös, A. Conzelmann, and R. Singer, Eds., pp. 227-248, Hofmann, Schorndorf, Germany, 1994. 

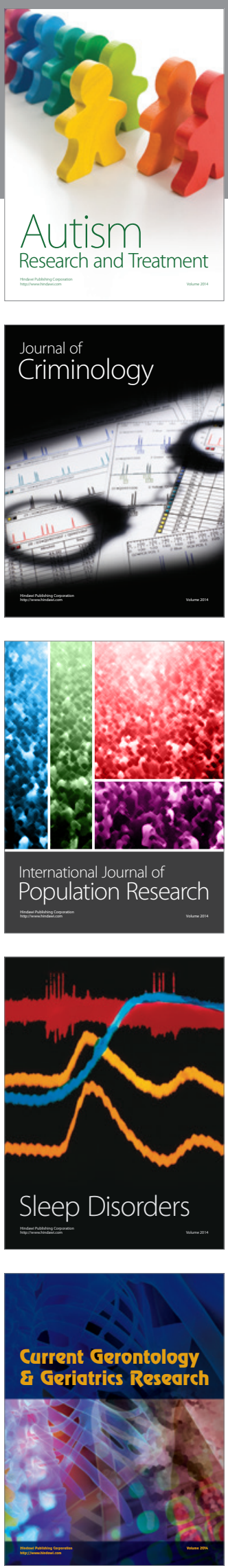
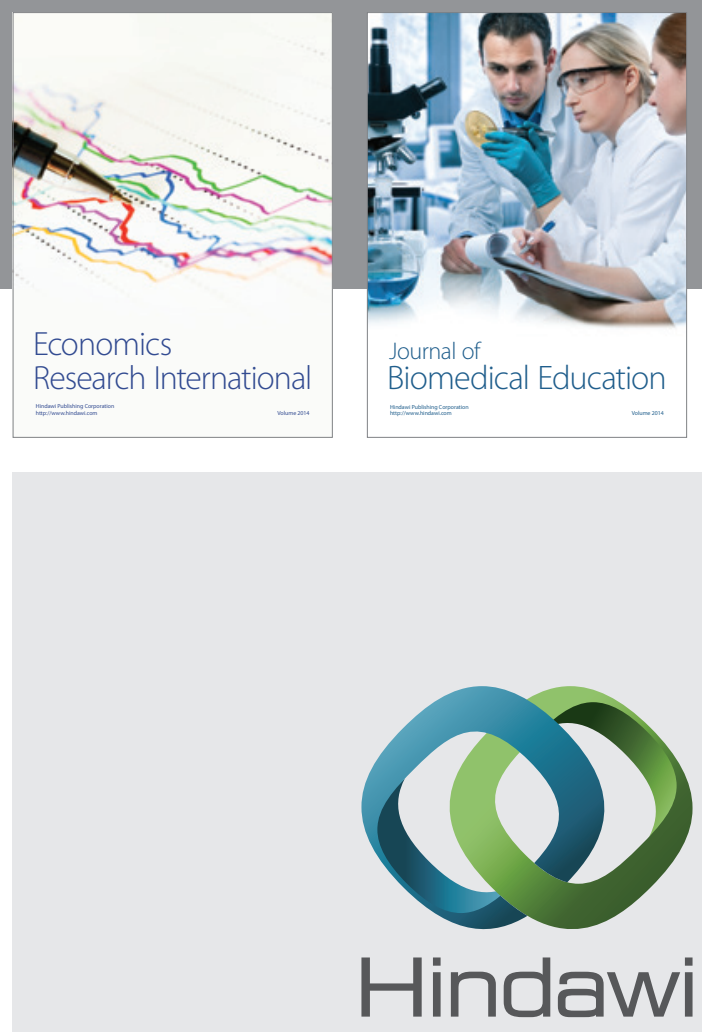

Submit your manuscripts at

http://www.hindawi.com
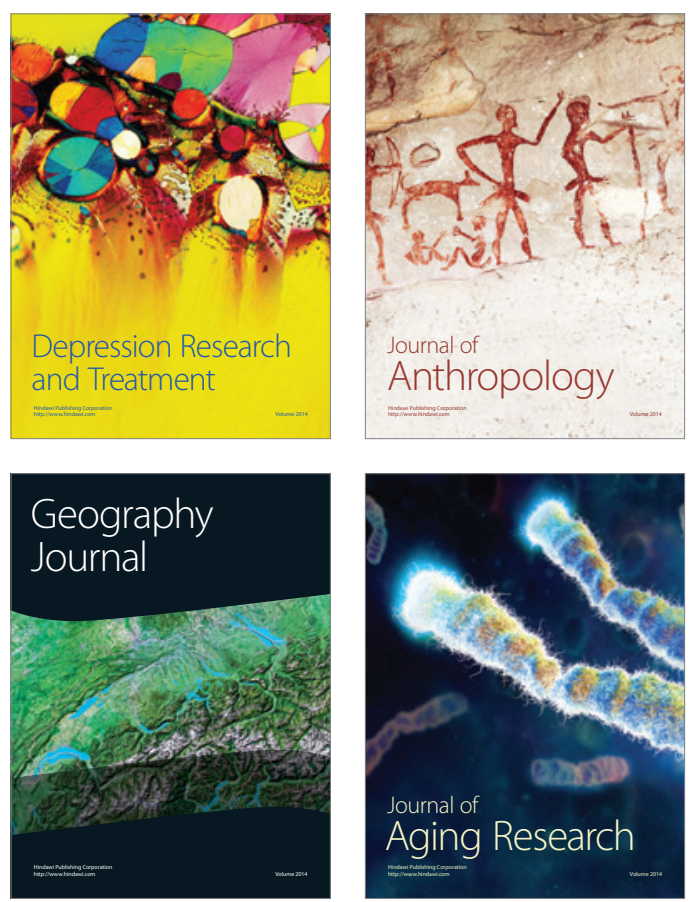
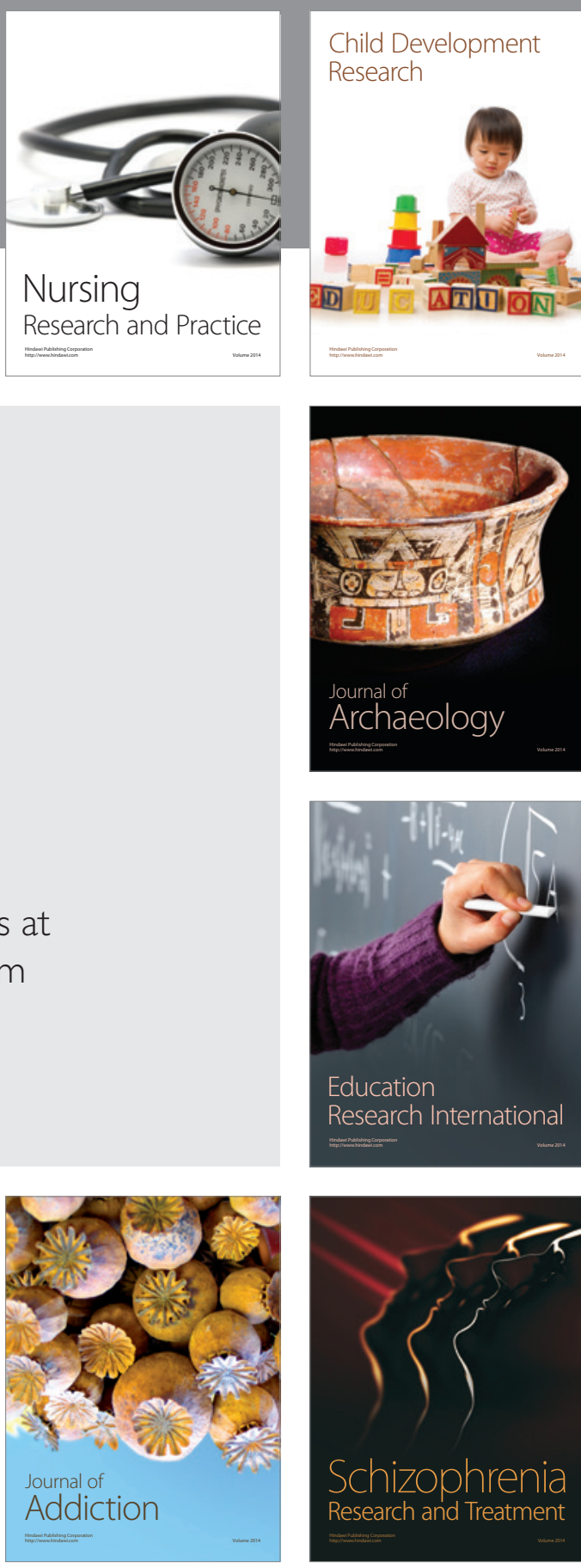

(D)
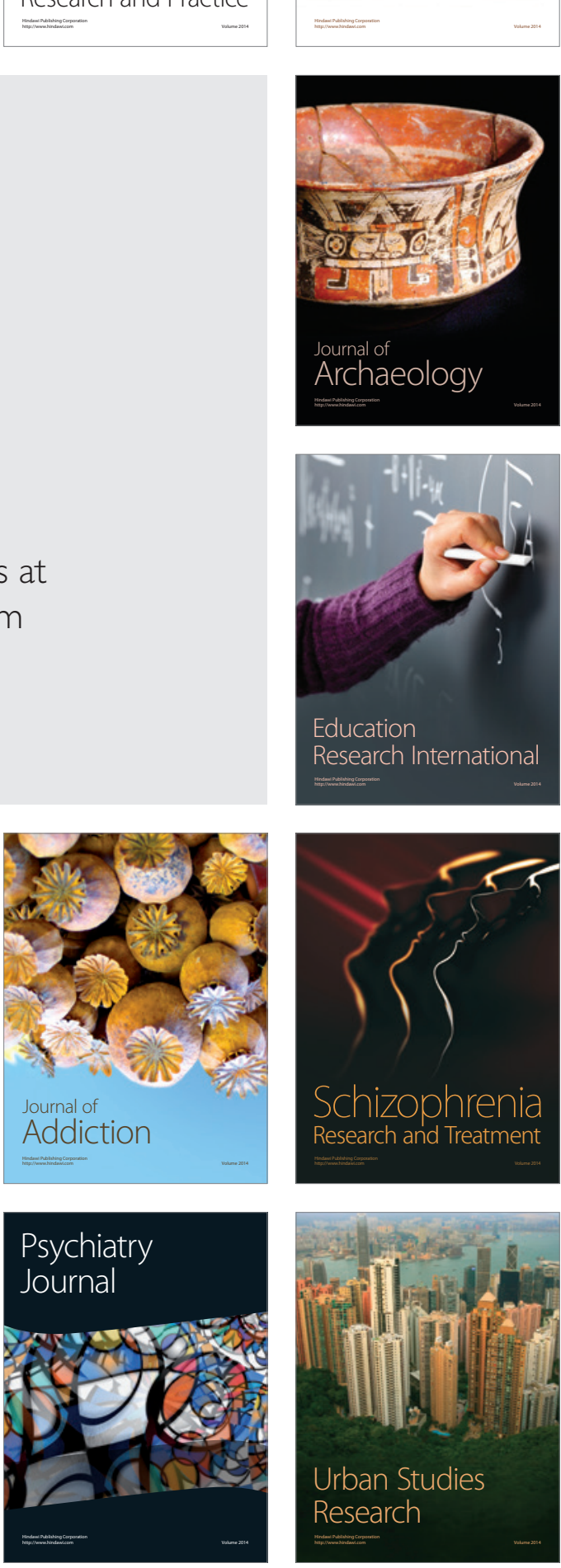